\title{
Time-Domain THz Near-Field Imaging Incorporating Hadamard Multiplexing Method
}

\author{
Mingguang Tuo ${ }^{1}$, Min Liang ${ }^{1}$, Jitao Zhang ${ }^{1,2}$, and Hao Xin ${ }^{1,3}$ \\ ${ }^{1}$ Department of Electrical and Computer Engineering, University of Arizona, Tucson, Arizona, 85721, USA \\ ${ }^{2}$ Fischell Department of Bioengineering, University of Maryland, College Park, Maryland, 20742, USA \\ ${ }^{3}$ Department of Physics, University of Arizona, Tucson, Arizona, 85721, USA
}

\begin{abstract}
Photoconductive antenna (PCA) array based $\mathrm{THz}$ near-field imager incorporating Hadamard multiplexing method is developed in this work. By using a $2 \times 2$ dipole antenna array as the THz transmitter, the system signal-to-noise ratio (SNR) is demonstrated to be improved by a factor of 2 as the theory predicts. Additionally, a 2-D scanning of a metallic structure on a THz-transparent substrate (with a total scanning area of $1 \times 1$ $\mathbf{m m}^{2}$ ) is experimentally implemented. Correlation coefficient estimation is made afterwards to quantify the reconstructed image quality.
\end{abstract}

\section{INTRODUCTION}

$\mathrm{T}$ ERAHERTZ (THz) near-field imaging system is developed to overcome the resolution limit for traditional $\mathrm{THz}$ far-field time-domain spectroscopy (THz-TDS). With the near-field setup, sub-wavelength spatial resolution could be achieved as reported in [1]. Different from a typical far-field THz-TDS system where the transmitter and receiver are attached onto $\mathrm{Si}$ lenses for focusing the $\mathrm{THz}$ wave, the near-field setup requires backside laser illumination to the antenna on a laser-transparent substrate. Therefore, no Si lens will be used, which induces the potential problem of lowering the system SNR.

In this work, a $2 \times 2$ dipole PCA array is adapted as the $\mathrm{THz}$ transmitter. The Hadamard multiplexing method [2] is then applied to demonstrate the improvement of the system SNR. 2-D scanning of a sample made of a metallic structure on GaAs-on-Sapphire substrate is implemented and analyzed.

\section{SNR IMPROVEMENT AND 2D SCANNING RESULTS}

The general setup of the near-field imager is shown in Fig. 1. First, the time domain responses of the four PCAs in the array are individually measured. Then, a Hadamard matrix is used to encode the bias signals of the four PCAs. The coded time domain responses of the four antennas are recorded with the same amount of measurement time. The single antenna responses can be decoded back from the coded measurement results by inverting the Hadamard matrix. The responses of the individually measured and the Hadamard decoded single antennas are plotted in Fig. 2(a) and 2(b). The decoded single antenna time domain responses are seen to be less noisy than the individually measured ones. A factor of 2 improvement of SNR is achieved by further quantifying the standard deviation

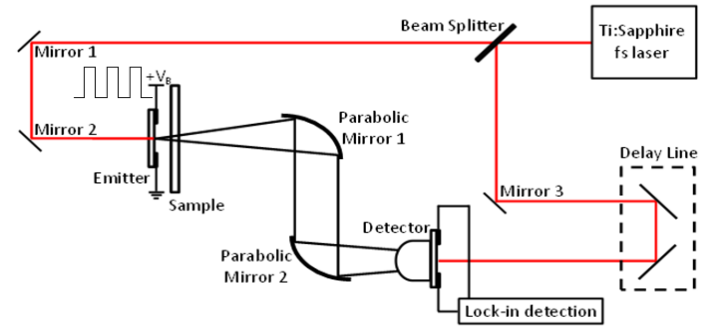

Fig. 1. General schematic of the near-field setup.
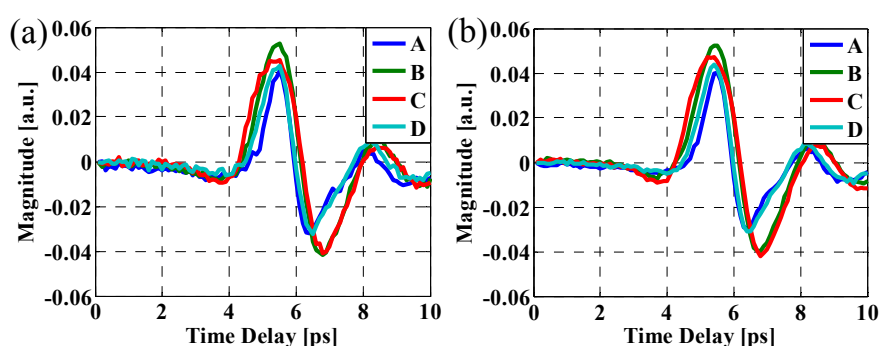

Fig. 2. The time domain responses of (a) the individually measured single antenna and (b) the Hadamard decoded single antenna.
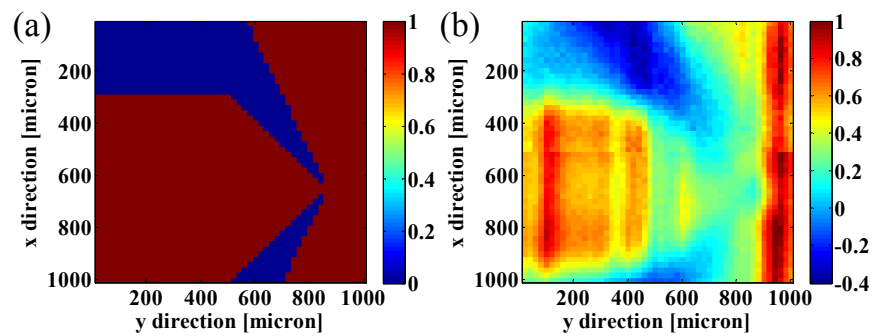

Fig. 3. (a) The digitized original structure image (1's amd 0 's are used to represent the substrate and metal areas, respectively) and (b) the reconstructed 2D scanning image by choosing a fixed time delay in the Hadamard decoded time domain responses.

based on multiple measurements for each scenario, which agrees well with theory.

In addition, a metallic structure fabricated on a GaAs-onSapphire substrate as shown in Fig. 3(a) is used as a sample for 2-D scanning. The total scanning area is $1 \times 1 \mathrm{~mm}^{2}$ (with scanning step of $20 \mu \mathrm{m}$ ) and each antenna in the array covers one quadrant of the total scanning area. By choosing a fixed time delay in the Hadamard decoded time domain responses, the normalized reconstructed image is given in Fig. 3 (b). The image quality is assessed by calculating the product-moment correlation coefficient between those two images [3]. A correlation coefficient of about 0.7 is obtained for the reconstructed image.

\section{SUMMARY}

A THz near-field imaging system using a $2 \times 2$ dipole PCA array and incorporating the Hadamard multiplexing method is realized in this work. As expected, SNR improvement of a factor of 2 is achieved. Moreover, for a sample of a metallic pattern on GaAs-on-Sapphire substrate, a correlation coefficient of about 0.7 representing the reconstructed image quality is obtained.

\section{REFERENCES}

[1] M. Tuo, J. Zhang, M. Liang, W.-R. Ng, M. E. Gehm, and H. Xin, "THz photoconductive antenna array based near field imaging," 40th International Conference on IRMMW-THz, Hong Kong, Aug. 23-28, 2015.

[2] M. Harwit. Hadamard transform optics. Elsevier, 2012.

[3] J. L. Rodgers, and W. A. Nicewander, "Thirteen ways to look at the correlation coefficient," The American Statistician, vol. 42, pp. 59-66, 1988. 